\title{
NATO-SCI 133 guides for planning and reporting tests of countermine equipment
}

\author{
Ric (H.M.A.) Schleijpen*, Hal Bertrand** \\ *TNO Defence, Security and Safety, P.O. Box 96864, 2509 JG The Hague, The Netherlands \\ ** Institute for Defense Analyses (IDA), 4850 Mark Center Drive, Alexandria, Virginia 22311, USA
}

\begin{abstract}
NATO Task group SCI-133 on "Countermine Technologies" under the NATO RTO (Systems Concepts and Integration) panel, has the goal to identify technologies for mine detection (close-in detection and remote detection) and mine neutralization (breaching, route clearing, area clearance) which provide the best short, medium and long-term potential in countermines operations. A brief overview of the activities will be given.

The main focus of the paper is on the work of the NATO RTO SCI-133 task group on test and evaluation procedures for landmine-detection/neutralization and mechanical clearance equipment. Interpreting test results in test reports, is often difficult due to incomplete descriptions of test procedures and lack of clear definitions of test parameters. Task group SCI-133 prepared a list of issues that should be addressed in test reports of landmine-detection equipment. These guidelines also give references to documents containing relevant definitions. The presentation is in the form of a checklist of questions to be answered when designing, conducting, and reporting on test and evaluation efforts.
\end{abstract}

Keywords: Countermine technologies, Land mine detection, Test and Evaluation procedures

\section{INTRODUCTION}

Task group SCI-133 "Countermine Technologies" under the NATO RTO SCI panel (Research and Technology Organisation, Systems Concepts and Integration), has the goal of identifying technologies for mine detection (close in detection and stand-off/remote detection) and mine neutralization (breaching, route clearing, area clearance) which show the best short, medium and long term potential for countermine operations. As a part of SCI-133's work, the group addressed testing and evaluation issues associated with countermine equipment.

The intention of this paper is to communicate the results of the NATO Task Group to a broader public than the NATO military community only. The work of the group will be published via the NATO channels. However, especially the work of the task group on the guidelines for test and evaluation of countermine equipment has a much larger group of potential users. We hope to reach users not only the military countermine community, but also the area of humanitarian demining.

It has been noticed during reviews of countermine programs of member nations that developmental and operational tests on similar, and sometimes the same, countermine problems are frequently repeated in more than one nation's test programs. Reasons commonly given for this were that the other country's test program did not address the same issues of interest, that certain test measurements were not taken, or that the available test data were not clear on what exactly was done. Therefore, SCI-133 undertook an effort to harmonize countermine test procedures and associated terminology. This resulted in a series of three "guides for planning and reporting test and evaluation activities" on systems and technologies for detection, mechanical demining, and non-mechanical neutralization systems. These guides are presented as the following parts of a NATO RTO document

- PART 1 - "Planning and Reporting Test and Evaluation Activities for Landmine and Minefield Detection"

- PART 2 - "Planning and Reporting Test and Evaluation Activities for Mine/Minefield Mechanical Demining Equipment"

- PART 3 - "Planning and Reporting Test and Evaluation Activities for Non-Mechanical Mine/Minefield Neutralization Equipment"

ric.schleijpen@tno.nl, phone +31 70374 0045, fax +31 70374 0654, www.tno.nl

Detection and Remediation Technologies for Mines and Minelike Targets XII

edited by Russell S. Harmon, J. Thomas Broach, John H. Holloway, Jr.

Proc. of SPIE Vol. 6553, 65531I, (2007) · 0277-786X/07/\$18 - doi: 10.1117/12.718597

Proc. of SPIE Vol. 6553 65531I-1 
To illustrate the content of the NATO work, this paper will discuss the guidelines for test and evaluation for landmine detection equipment as an example.

Each PART is intended as a guide of what should be done in a test program rather than a test standard of what must be done. Therefore, each guide takes the form of a list or checklist of suggestions of what to do, how to do it, and how to report on test activities. To encourage the use of the guides, they were formatted to address test issues and checklist items in the order that they most likely would be encountered in the development of a test or test program. While the checklist details vary from guide to guide, the sequence of the general issue areas addressed is the same in each guide.

This check-list is not a detailed "cook-book" for planning and reporting test and evaluation activities. Also, it is beyond the scope of the check-list to list definitions of all the parameters describing test conditions and results. Although definitions are not prescribed by this document, definitions should be given unambiguously in the test reports or references to appropriate definitions in other documents should be given. Some references to documents containing useful definitions are given in this document. The use of proper and commonly used definitions of terms and figures of merit will make test reports more useful to a larger community.

Each test guide was developed through an iterative process of reviewing tests standards, technical input by experts, and consensus building on changes and additions. Because of the detail for testing and evaluation in the three technology areas differ quite significantly, each guide was developed for use as a stand-alone document. The full documents will be published by NATO RTO.

\section{STATEMENT OF TEST OBJECTIVES}

The first issue to be addressed in the design phase of a test is the specification of the test objective(s) and the type of test. The test objective(s) should be clearly stated and explained in the test plan, or simply said: What are the key question(s) to be answered with the help of the test results? For example typical questions could be:

- Can the equipment do, at this phase of its development cycle, what it is designed to do?

- Under what environmental conditions is it expected to function and can it perform under the conditions of the test?

- Does the equipment meet the performance objectives required to continue the system's development or satisfy the endpoint requirements of the current phase?

These questions should also be included and explained in the test report. Clear criteria for answering these questions with yes or no should be defined using the guidelines of this document. A test can therefore be called successful when these questions can be answered, even when the answer is negative or when the system fails to meet the performance requirement.

Also the type of test should be explained. For example:

- Is it a comparative test between two or more sensors and systems?

- Is it a test to explore the limits of the detection capabilities of one sensor?

- Is the test designed to evaluate a new detection concept?

- Is the test set up to determine whether a more mature detection system design can meet its baseline performance requirements?

- Is the test a formal qualification test of a system by verifying that it meets all specified requirements?

\section{DESCRIPTION OF SYSTEM UNDER TEST}

\section{Description of detection principle}

The second issue to be addressed is the description of the system under test and the status or condition of the system during the test. The report should give an explanation of the detection principle, the technology, and the method of operation of the system under test.

\section{System description}

After that, a description of the specific system should be given including the details of the manufacturer, commercial name and model number or other designation. Including photographs and drawings in the test report will contribute to a better understanding of the system under test. 
An indication of the Technology Readiness Level (TRL) (see reference 1) of the system should be given on the system level and, when applicable, on component level.

A detailed description of the system should be given at the major component level (signal source, detector, data processing, and platform if applicable). This should include the operating conditions and procedures needed to achieve the design performance specifications of the system. Any known performance limitations should be indicated.

A detailed system description may include the following items. It is recognized that many of the description items below will be verified (if design goals) or quantified (if unknown) by the intended test.

- Dimensions of equipment (or components).

- Description of the system hardware/software and its operation.

- Description of the sensor platform (if applicable).

- Operational issues related to the equipment under test.

\section{TEST DESIGN}

The test should be designed to answer the key questions stated in the test objective(s). The starting points for test design may include:

- The scenario in which the system will be used

- The requirements as defined in a procurement process if applicable

- The perspective of the user of test results, who may be:

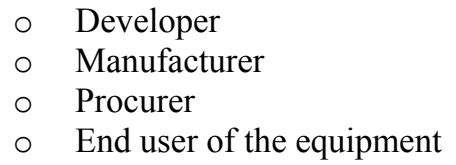

The degree of control in the test should be discussed. The parameters that are controlled should be listed and discussed. For the parameters that cannot be controlled, it should be stated whether or not they are measured. In each case provide clear reasons why a parameter is treated in a certain way. In general, one can discriminate the following types of tests as having decreasing levels of control:

- Laboratory tests under stable indoor conditions

- Outdoor tests under controlled conditions

- Outdoor test without controlled conditions

- Operational or field tests in real mine fields

Apart from the detection performance of a detection system, some other issues may need testing as well. These issues, strictly speaking, are not part of a detection performance test but may influence the detection performance. Examples of these issues are:

- System robustness

- System logistics

- Human factors (e.g. man machine interface)

- Environmental limitations

- Electromagnetic Interference/Electromagnetic Compatibility (EMI/EMC)

\section{Number of test objects}

The test philosophy can be to test a system's performance against a large number of identical/similar test targets. It turns out that seemingly "large" (but manageable) numbers of targets are still quite small in relation to the required statistical reliability needed in mine detection performance analysis. In this respect it is necessary to discuss the statistical significance of tests. This subject is treated in references 2 to 5 .

An alternative test philosophy is to test against a smaller number of targets with an increasing degree of difficulty of detection. For example in a comparative test one could bury a number of identical targets at increasing depth and compare the results.

Limited availability of test targets, like mines, or limited resources may limit the number of targets used, but the consequences on the statistical significance of the test should be considered in the interpretation of results. 


\section{TEST TARGETS AND DEPLOYMENT EMENT OF TEST OBJECTIVES}

The test targets and their deployment should be described. For targets the following issues should be addressed.

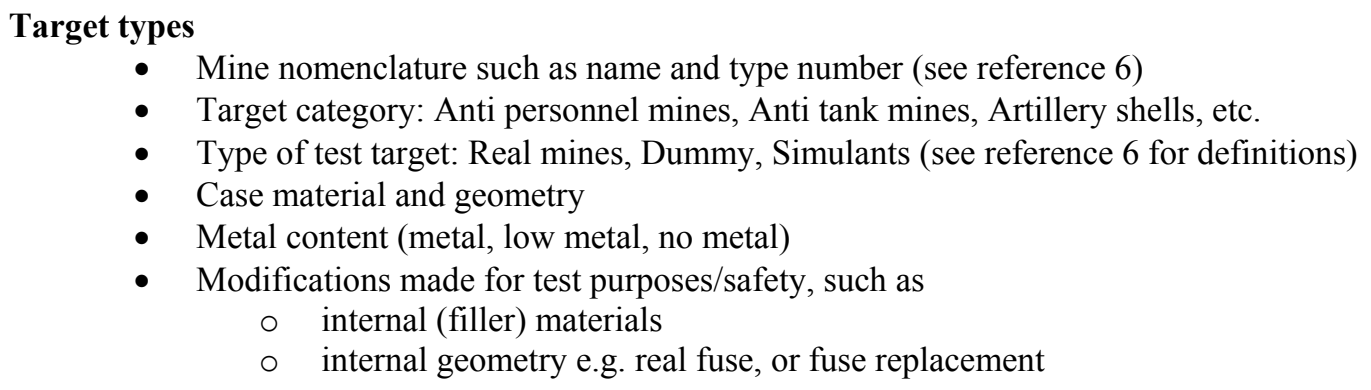

Target deployment (see reference 7).

- Burial depth

- Orientation

- Number of targets

- Density of targets / separation between targets

- Maps and pictures

\section{Emplacement technique}

- Describe the burial method, e.g. use of military equipment, burial by hand, etc.

- Describe the way the soil is disturbed.

- Describe to what extent soil disturbance has disappeared by weathering or aging (time between target placement and execution of the test).

\section{Non-mine objects}

It is very common in detection tests to insert objects which give mine-like responses to sensors in the test area. For example pieces of metal of sufficient size will cause a metal detector to respond even if the metal is not part of a mine. This kind of "clutter" objects and other sources which generate false alarms are discussed in more detail in the section on test environment. Intentional emplacement of objects which generate mine-like responses will allow testing the capability of a sensor to discriminate between real targets and other objects. In such cases, the description of non-mine test targets should be at the same level of detail as given for the real mine targets or surrogate mine test targets. The emplacement of non-mine objects is very likely to generate a number of incorrect detections which may not occur under natural conditions, which must be reflected in the (statistical) interpretation of the results.

\section{TEST ENVIRONMENT}

\section{Soil properties}

Depending on the technology or operating principle of the detection system under test, different soil properties will be relevant. The first step is to characterize the soil into categories of clay, sand, loam, silt, gravel or stone, or mixtures. This characterizes the soil by ratios of particle size classes (see references 8-10). The soil type, together with a number of secondary parameters that are influenced by the weather, temperature, moisture and water table, will determine the soil properties which are relevant for various detection technologies. An overview of these soil properties is given in table 1. (based on reference 11). This list has been put together from a general knowledge of the working principles of the various techniques and makes no claim as to the relative influence of the various soil properties identified. In each case a detailed consideration and analysis of the properties influencing performance should be done to identify factors with significant influence on a detection technology. Table 1 is meant to promote thoughtful consideration of properties and to avoid ad hoc measurements which are often made. 
Table 1: Landmine detection technologies and relevant soil properties

\begin{tabular}{|l|l|}
\hline Detection technology & Relevant soil properties \\
\hline Magnetostatics & Magnetic susceptibility \\
\hline Electrical Impedance Tomography & Electrical conductivity \\
\hline Electromagnetic Induction & Magnetic susceptibility \\
& Electrical conductivity \\
\hline Nuclear Quadrupole Resonance & Piezo-electric materials (e.g. quartz contents) \\
& Magnetostrictive materials \\
& Electrical conductivity \\
& Magnetic susceptibility \\
& Electrical permittivity \\
\hline Active and Passive Microwaves & Electrical permittivity \\
including Radar & Electrical conductivity \\
& Magnetic susceptibility \\
\hline Infrared & Moisture content \\
\hline Thermal emissivity \\
& Thermal resistivity \\
& Thermal diffusivity \\
& Specific heat capacity \\
\hline Neutron Interrogation & Moisture content \\
\hline X-ray backscatter & Elemental composition \\
\hline Trace Explosives Detection & Elemental composition \\
& Effective density \\
\hline Prodders (including instrumented) & Soil porosity \\
& Moisture content \\
& Precipitation history \\
& Cation exchange capacity \\
& Other chemicals and parameters affecting \\
& Absorption and adsorption of explosives \\
\hline Acoustic / Seismic techniques & Bulk density \\
& Grain size distribution, composition \\
& Elastic modulus \\
& Moisture content (versus depth) \\
\hline
\end{tabular}

A description of the method(s) used to measure soil parameters should be given, e.g.:

- Measurement instruments used

- Method of taking samples

- Number of samples

- Depth at which samples are taken

- Distribution of samples over the test area

Electromagnetic soil parameters should be measured in the specific spectral range relevant for the detection technique under test. Frequency dependence of these parameters may influence the test results. Efforts are ongoing to derive these soil parameters directly from the chemical composition, texture and structure of the soil.

\section{Variability, gradients and non-homogeneity of soil properties}

Even if the relevant soil properties can be measured, one must bear in mind that soil properties will very likely show spatial and temporal variations. Soil properties may change predictably with depth, but can also have local nonhomogeneities or anomalies, and interfaces between adjacent soil types. Temporal changes are induced by seasonal, 
diurnal and weather variations. Weather, especially, will cause variations in soil properties which depend on temperature, moisture and/or water table. Even the weather conditions are constant, evaporation will cause changes in soil moisture content.

\section{Atmospheric conditions}

Ambient air properties may also influence the test result(s), depending on the principle of the detection technique. For example: strong atmospheric transmission losses will affect the performance of electro-optical detection techniques. Weather conditions can be characterized by: temperature, solar irradiance, humidity, air pressure, cloud cover, wind speed, and wind direction. Also, variations of these conditions prior to the test may be relevant. For example soils with a large heat capacity will not immediately follow rapid changes is solar irradiation and ambient temperature.

\section{Other sources of performance degradation}

Other environmental issues may influence the performance of detection systems, e.g.:

- Surface roughness

- Vegetation in the test area. Photographs of the vegetation are a good addition or alternative for narrative characterization of the vegetation.

- Electromagnetic interference from man-made sources e.g. radio emissions or power lines or natural sources e.g. thunderstorms.

\section{Sources of non-target detections}

Detections indicated by the system under test, that cannot be attributed to test targets, can originate from other sources in the test environment. Together with the environmental parameters described above, these sources determine the background clutter in a test.

- Intentionally positioned alarm generating objects

- A test could involve placing some non-target objects known to cause false alarms. This is usually done when determining the ability of the detector to discriminate between valid real targets and other target-like objects. These non-target objects should be described to the same level of detail as are the mine targets. As mentioned in the section on test targets, information on these deliberate false alarm sources is critical for interpreting test results.

- Non-intentional anomalies

- Even in the absence of targets or intentionally positioned alarm generating objects, the soil and/or environment can yield detection responses by the system under test. The specific origin of these false alarms is usually not known to the test designer and it may be difficult to give a clear description of the anomalies. Often one is not even aware of their presence before the test. Therefore, whenever possible, it is desirable to characterize the clutter of the soil in the test area before (or after) placing the test targets. This can be done, for example by scanning the test area with a detector that uses the same detection principle as the system under test and that has similar or better detection performance.

Characterizing clutter (e.g. by establishing a probability density function) during the tests is very useful and should be repeated since clutter conditions may change during testing. Some sources of clutter could be:

- Cavities in the soil

- Water patches (in the soil, on the surface)

- Man-made objects, lost tools, keys, cans

- Lumps of clay or organic matter

\section{Reference and calibration objects}

Reference objects enable one to relate test data to known standards. They can be used for a number of purposes. In each case the reference object should be well described. When widely accepted standard reference objects exist, these should be used for the test when available. 
- Measuring the detector response of reference objects, with similar physical characteristics as the mines to be detected can help to characterize the test area, the soil, the environment, and detector response. Physical characteristics of these reference objects should be well defined. It is also desirable to define some basic measurements on these reference objects and to describe the kind of information these measurements will provide.

- Measuring the response of a sensor to a specific reference object that has been used in other tests with other sensors, may help to interpret and compare the results of different tests.

- Reference objects can be used to calibrate or recalibrate sensors before and during tests (when this is a part of the test procedure).

The use of reference objects (or fiducials) on known locations, will give references in the spatial domain, which will help to characterize the locational accuracy or precision of detections.

\section{TEST PROCEDURES}

Test results will depend not only on intrinsic sensor parameters, but also on how the sensor is operated during tests. The description of how the sensor is operated should be part of the test procedures.

\section{Sensor operation}

The way a sensor is operated in a test may differ from how the end user is expected to use it. This difference may be intentional or un-intentional and may change over the test period. A number of issues related to sensor operation that can affect its performance are mentioned below:

- Calibration, Monitoring calibration stability

- Tuning of threshold levels to local conditions

- Altitude above ground / Stand off

- Speed of scan / Static / Measurement time

- Number of scans / Scan pattern

The calibration procedure and criteria for recalibration during the test should be well described, since calibration effects will directly influence test results.

\section{Role of the operator}

Some detection systems need a man in the loop. This may be for interpretation of sensor signals or for controlling sensor motion (or both). In either case the condition of the operator may influence test results. This will depend among other factors on:

- Training

- Motivation

- Experience

- Physical condition (Hearing, Eyesight, Fatigue etc.)

- Operator burdens for remote control

It should be noted that the condition of the operator is likely to change during the test. Also the degree of influence of the operator on the test results should be considered in the context of the test and be noted in the report.

\section{Data recording}

The test report should describe all measured quantities and parameters, how the data was recorded, and all data recorded during the test. This should also include a description of how the sensor position was measured during the test and the method used for this measurement. 


\section{MEASURES OF EFFECTIVENESS}

Measures of Effectiveness (MOE) must be defined in order to answer the key question(s) related to overall test objective(s). There exists a confusing array of terminology used by various test entities to describe test results. This frequently prevents interpretation and easy comparison of results between tests. To avoid this confusion all MOEs have to be clearly defined, as well as all terms used to describe MOEs. As mentioned before, definitions are not prescribed by this document. However, definitions should be given unambiguously in the test reports or explained by referring to appropriate definitions in other documents (see references 12,13). In the case a test is designed to determine whether a detection system meets a specific performance level (expressed in a well defined MOE), the specific threshold level should be mentioned in the report. It can be useful to the reader to discuss the motivation to choose this particular threshold level.

For an unambiguous MOE for a detection system it should be clearly defined what is meant by detection (and classification and/or identification when applicable)

In general the optimization of a detection process often involves one or more trade offs. For example, between minimizing the number of missed (undetected) mine targets and minimizing the number detector signals incorrectly interpreted as a mine target. Therefore, definition of MOEs should at least include:

- number of correctly detected mine targets

- number of undetected (missed) mine targets

- number of false alarms (detector signals incorrectly interpreted as a mine target)

The number of false alarms may be split in false alarms due to intentionally placed objects and false alarms due to other anomalies (see the section on sources of non-target detections).

In some system tests results of intermediate steps of signal processing may be available. Mine targets which remain undeclared in the final output, may have been detected initially but rejected during signal processing. In this case evaluation of intermediate results may be useful, to gain insight into possible methods to improve signal processing. In a broader sense, MOEs can involve the following terms:

Target detection

- (Rate or probability of) Detection (of test targets)

- (Rate or probability of) Classification

- (Rate or probability of) Identification

- (Probability of) Discrimination between two test targets

False alarms and unwanted alarms

- False alarm rate (define rate e.g. per unit area)

- Area related to the alarm or detection (see also: Halo of detection)

- Area of test site

- Clutter level

Target localization:

- Accuracy of: Y,Y Position, Depth

- Marking accuracy

- "Halo" of the detection, i.e. maximum radius of the declared mine location to the nearest actual edge of the mine target (determined among others by sensor footprint, target size, marking accuracy etc.)

Robustness of detection

- Sensitivity of test results with respect to the parameters which have been changed during the test

- Margin of detection: e.g., comparison of the signal-to-noise ratio (or signal-to-clutter ratio) to the threshold level for detection

Measurement duration

- Reports total measurement time (usually expressed in $\mathrm{m}^{2} /$ hour) and directly affects the expected mission rate of advance 
Often Receiver Operating Characteristics (ROC) are used to present the performance of a sensor. For a clear interpretation of results one should describe how the ROC curves are generated and which parameters are varied over the set of curves. ROC curves can be used, for example, to show the effect of variation of a threshold level in the system under test. The ROC curve then illustrates the relation between the number of correctly detected mine targets and the number of false detections as a function of this threshold level.

\section{ANALYSIS AND RESULTS}

\section{Analysis}

All analyses performed to answer the key question(s) related to the test objective(s) should be described, including all terms and methods used. To increase the value of a test report for others who wish to "translate" test results for their own needs, it is very helpful to give intermediate results and possibly some examples of raw data. Graphs should be included where applicable.

The analysis may involve:

- Raw data (raw sensor signal)

- Processed data

- False alarms

- Likely sources of false alarms

- Features of false alarms (features bearing similarities to targets)

- Sensitivity to operator performance

- Comparison of the results/detector signals to predictions and models

Features of targets which are measured by the sensor may be analyzed. These features may be used in the decision process whether or not to declare a mine detection. Examples of features include:

- Depth

- Size

- Volume, e.g. the target's geometrical size/volume or the signal's response size/volume

- Shape and geometry, e.g. symmetric vs. asymmetric

\section{Scoring}

The analyses will result in a scoring metric. For this purpose the data is transformed into detections for comparison with ground truth (known location of test targets). Correlating the detections with the ground truth (taking into account the detection area defined as target area plus halo) will result in the number of correct detections. Some test facilities hold ground truth as confidential information and have an independent referee establish the score. This allows repeated use of a test site for blind tests, without having to constantly change the target locations. On the other hand it does not allow giving a complete feedback to the system developer. In some cases calibration lanes are provided with the ground truth for the mine targets in those lanes, to allow for system performance checks prior to a formal test.

In view of the relatively low number of targets usually involved in tests, it is desirable to present the results in terms of numbers of targets detected out of the total number of targets present, rather than a detection percentage. When system performance is expressed in probability of detection also confidence intervals should be given.

\section{Discussion of Results}

Describe how well the interpretation and validity of test results addresses the test objective(s) adopted in the test plan.

\section{Lessons learned}

A section on lessons learned during the test will enhance the value of the test report for a wider community. 


\section{CONCLUSIONS AND RECOMMENDATIONS}

Test report conclusions should state the answer(s) to key question(s) previously stated and compare the results to the objective(s) previously stated.

A section presenting recommendations that flow from the test program should be provided. Recommendations can be made in a number of areas. Areas for which recommendations have been frequently presented are listed here:

- Equipment design

- Data processing

- Operational procedures

- Training programs

- Shipping and transportation procedures

\section{GENERAL COMMENTS ON THE GUIDELINES}

This paper presented he guidelines on planning and reporting test and evaluation of countermine equipment as set up by the NATO RTO SCI-133 task group. As an example the guidelines for test and evaluation of landmine detection are discussed. These guidelines are a contribution to improved reports and papers on tests of countermine equipment. The use of these guidelines should extend the use of reports and papers to a larger community, because interpretation of the results will be made easier for non-participants in the test.

We would like to conclude with some general comments:

- Feedback from use of the guidelines - An update of these guides based on feedback from users would be a worthwhile endeavour for a future NATO-SCI working group.

- Finding new technologies - Opportunities for finding new countermine systems will also come as the result of technology transfer from other fields. For this reason, reports should clearly state shortcomings of equipment under test.

- For systems under development - Multinational cooperative efforts in test and evaluation should be undertaken to shorten the time involved, to spread cost amongst participants, and to avoid duplication.

\section{ACKNOWLEDGEMENT}

Contributions to this document were made by: E. den Breejen, W. de Jong, J. Rhebergen, A. Schoolderman (TNO Defence, Security and Safety). L. Merlat (Institut Saint Louis, St Louis, France), Y. Das (DRDC, Suffield, Canada), N.Wright (U.S. Army RDECOM CERDEC, Fort Belvoir, US), B.Haugsted (DDRE, Copenhagen, Denmark) and other SCI-133 members.

\section{REFERENCES}

[1] John C. Mankins, “Technology Readiness Levels: A White Paper,” NASA, 1995, http://www.hg.nasa.gov/office/codeq/trl/trl.pdf.

[2] Katherine M. Simonson, "Statistical Considerations in Designing Tests of Mine Detection Systems: I - Measures Related to the Probability of Detection," Sandia National Laboratories, SAND98-1769/1, August 1998.

[3] Katherine M. Simonson, "Statistical Considerations in Designing Tests of Mine Detection Systems: II - Measures Related to the False Alarm Rate," Sandia National Laboratories, SAND98-1769/2, August 1998.

[4] R. Voles, "Confidence in the Assessment and Use Mine Detection Systems," in IEE editor, "The Detection of Abandoned Landmines," p 28, Volume 458 of Conference Publications, IEE, Oct 1998.

[5] Anne M. Andrews, Vivian George, Thomas W. Altshuler, "Quantifying Performance of Mine Detectors With Fewer Than 10,000 Targets" in Detection and Remediation Technologies for Mines and Minelike Targets II, SPIE conference 3079, p. 273, 1997.

[6] STANAG 4587, "Close-in Landmine Detection Test Procedures," (in the process of ratification) 
[7] CEN Workshop Agreement, CWA 14747: Test and Evaluation - Metal Detectors, June 2003.

[8] J. Hendrickx, R.L. van Dam, B. Borchers, J. Curtis, H.A. Lensen, R. Harmon, "Worldwide Distribution of Soil Dielectric and Thermal Properties," in Detection and Remediation Technologies for Mines and Minelike Targets VIII, SPIE conference 5089, p. 1158, 2003.

[9] British Soil Classification System: see following website for a discussion of soil classification: http://fbe.uwe.ac.uk/public/geocal/SoilMech/classification/soilclas.htm (December 2005)

[10] American Society for Testing and Materials (ASTM) International, D2487-00 Standard Classification of Soils for Engineering Purposes (Unified Soil Classification System),10 Mar 2000 (12 pages), see following website to purchase or use alternate sources: http://www.astm.org

[11] Y. Das, J. McFee, K. Russel, G. Cross, T.J. Katsube, "Soil Information Requirements for Humanitarian Demining: The Case for a Soil properties Database," in Detection and Remediation Technologies for Mines and Minelike Targets VIII, SPIE conference 5089, p. 1146, 2003.

[12] D. Borghys, L. Sevigny, R. Gabler, J. Blanc-Talon, P.B.W. Schwering, H.C. Palm, J.F. Haddon, N. Nasrabadi, "Multisensor Image Exploitation", Final Report of SET-022/TG10, RTO-TM-011 AC/323(SET-022)TP/10, 2002

[13] J.M. Cruickshank, J. Maheux, R. Gabler, K. Bers, J. Hilger, C. Walters, "Performance Measurement of MultiSensor Fusion Systems", Final Report of SET-070/RTG.38, RTO Technical Report, 2005. 